\title{
Variability in the CoRoT photometry of three hot O-type stars
}

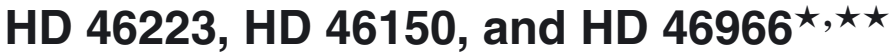

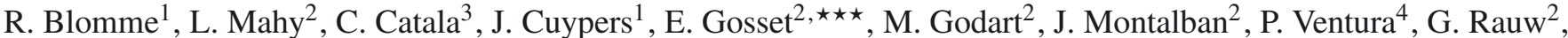

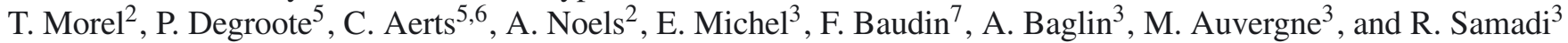 \\ 1 Royal Observatory of Belgium, Ringlaan 3, 1180 Brussel, Belgium \\ e-mail: Ronny.Blomme@oma.be \\ 2 Institut d'Astrophysique et de Géophysique, University of Liège, Bât. B5C, Allée du 6 Août 17, 4000 Liège, Belgium \\ 3 LESIA, UMR 8109, Observatoire de Paris, 5 place Jules Janssen, 92195 Meudon Cedex, France \\ ${ }^{4}$ INAF, Osservatorio Astronomico di Roma, via Frascati 33, 00040 Monteporzio Catone (Roma), Italy \\ 5 Instituut voor Sterrenkunde, K.U. Leuven, Celestijnenlaan 200D, 3001 Leuven, Belgium \\ 6 Department of Astrophysics, IMAPP, University of Nijmegen, PO Box 9010, 6500 GL Nijmegen, The Netherlands \\ 7 Institut d'Astrophysique Spatiale (IAS), Bâtiment 121, Université Paris-Sud, 91405 Orsay Cedex, France
}

Received 24 March 2011 / Accepted 29 June 2011

\section{ABSTRACT}

\begin{abstract}
Context. The detection of pulsational frequencies in stellar photometry is required as input for asteroseismological modelling. The second short run (SRa02) of the CoRoT mission has provided photometric data of unprecedented quality and time-coverage for a number of O-type stars.

Aims. We analyse the CoRoT data corresponding to three hot O-type stars, describing the properties of their light curves and search for pulsational frequencies, which we then compare to theoretical model predictions.

Methods. We determine the amplitude spectrum of the data, using the Lomb-Scargle and a multifrequency HMM-like technique. Frequencies are extracted by prewhitening, and their significance is evaluated under the assumption that the light curve is dominated by red noise. We search for harmonics, linear combinations, and regular spacings among these frequencies. We use simulations with the same time sampling as the data as a powerful tool to judge the significance of our results. From the theoretical point of view, we use the MAD non-adiabatic pulsation code to determine the expected frequencies of excited modes.

Results. A substantial number of frequencies is listed, but none can be convincingly identified as being connected to pulsations. The amplitude spectrum is dominated by red noise. Theoretical modelling shows that all three O-type stars can have excited modes, but the relation between the theoretical frequencies and the observed spectrum is not obvious.

Conclusions. The dominant red noise component in the hot O-type stars studied here clearly points to a different origin than the pulsations seen in cooler $\mathrm{O}$ stars. The physical cause of this red noise is unclear, but we speculate on the possibility of sub-surface convection, granulation, or stellar wind inhomogeneities being responsible.
\end{abstract}

Key words. stars: variables: general - stars: early-type - stars: oscillations - stars: individual: HD 46223 - stars: individual: HD 46150 - stars: individual: HD 46966

\section{Introduction}

Even though the O-type stars are located in the HertzsprungRussell (HR) diagram inside a zone where pulsations are expected, no asteroseismological modelling of these stars could be performed prior to the CoRoT (Convection, Rotation and planetary Transits, Baglin et al. 2006; Auvergne et al. 2009) mission. Indeed, few examples of variability have been identified. The most obvious ones are the $09.5 \mathrm{~V}$ stars $\zeta$ Oph (Kambe et al. 1997) and HD 93521 (Howarth \& Reid 1993; Rauw et al. 2008), whose variability is likely related to non-radial pulsations with periods of a few hours. However, the majority of these detections

* The CoRoT space mission was developed and is operated by the French space agency CNES, with participation of ESA's RSSD and Science Programmes, Austria, Belgium, Brazil, Germany and Spain.

$\star \star$ Tables 2-4 are available at the CDS via anonymous ftp to cdsarc.u-strasbg.fr (130.79.128.5) or via

http://cdsarc.u-strasbg.fr/viz-bin/qcat?J/A+A/533/A4

$\star \star \star$ Senior Research Associate F.R.S.-FNRS. were made spectroscopically. The reason is that the amplitudes of the pulsations are too low to be measured from ground-based photometry. Furthermore, these pulsations can be contaminated by variable stellar winds.

The second short run (SRa02) of the CoRoT satellite in the asteroseismologic channel was partly devoted to the investigation of the photometric variability of O-type stars. Pointing towards the anti-centre of the Galaxy, this instrument observed objects belonging to the young open cluster NGC 2244 inside the Rosette nebula and to the surrounding association Mon OB2. The CoRoT data of three O-type stars that are part of this run have been analysed in previous papers: HD 46149 by Degroote et al. (2010), HD 47129 by Mahy et al. (2011), and HD 46202 by Briquet et al. (2011), the latter paper containing the only forward modelling in terms of seismic data of an $\mathrm{O}$ star so far.

In the present paper we study the three remaining O-type stars that are part of the SRa02 run. One of the targets is HD 46223, the hottest member of NGC 2244. Situated at about 1.4-1.7 kpc (Hensberge et al. 2000), this star was previously 
quoted in the literature as having a spectral type O5 V (Hiltner 1956; Bisiacchi et al. 1982), O5 ((f)) (Conti \& Leep 1974), O4 ((f)) (Massey et al. 1995) and O4 V((f+)) (Mahy et al. 2009). The nine-year spectroscopic campaign by Mahy et al. to investigate multiplicity did not reveal any significant variation of the radial velocity related to the presence of a companion. In addition, Chandra mosaic observations of NGC 2244 (Wang et al. 2008) detect no strong clustering of X-ray sources around HD 46223, leading to the conclusion that there is also no lower-mass neighbour present. According to Wang et al., the small number of close-by X-ray sources could indicate that this star is younger compared to the population of the central part of the cluster.

The next target is HD 46150, the second hottest star in NGC 2244. Literature values for the spectral type are between O5 V((f)) (Underhill \& Gilroy 1990; Maíz-Apellániz et al. 2004) and O5.5 V((f)) (Conti \& Ebbets 1977; Garmany et al. 1980). The detailed study of Mahy et al. (2009) confirms the O5.5 V((f)) spectral type. Radial velocity variations have been detected in this star, but it is not clear if these are caused by binarity or motions in the stellar atmosphere (see Mahy et al. 2009, and references therein). Mahy et al. monitored this star but could not find any significant period in their data. They note the lack of spectral variability over short timescales ( $\sim 7$ days).

The third target studied in the present paper is the late O-type star HD 46966, belonging to the Mon OB2 association. The spectral classification generally agrees on O8.5 V (Conti et al. 1977; Garmany et al. 1980; Mahy et al. 2009) or O8 V (Munari \& Tomasella 1999; Maíz-Apellániz et al. 2004). Moreover, spectroscopic monitoring reveals no clues on the existence of a secondary component (Mahy et al. 2009).

These three stars are among the youngest (1-6 Myr, Bonatto $\&$ Bica 2009) and most massive observed by the CoRoT satellite. They therefore have the potential to extend our knowledge about pulsations into the higher-mass domain. The CoRoT light curves are of unprecedented quality and have a long observational time span (longer than the rotational cycle). They thus provide the ideal data set to reveal the presence or absence of such pulsations.

We first present in Sect. 2 the detailed frequency analysis of the HD 46223 CoRoT light curve. In Sects. 3 and 4 we give the frequency analysis of HD 46150 and HD 46966 respectively. Section 5 is devoted to determining the theoretically expected pulsation frequencies. We discuss the results in Sects. 6, and 7 we provide the conclusions of our research.

\section{HD 46223}

\subsection{CoRoT data}

The data we used here were obtained during the CoRoT second short run (SRa02) made from 08 Oct. to 12 Nov. 2008. We started from the level N2 data (Samadi et al. 2007), which for each observation list the time, flux, error on the flux, and flagging information. The flagging indicates whether these data are contaminated by instrumental and environmental conditions of the CoRoT satellite such as, e.g., the South Atlantic Anomaly (SAA) and other Earth orbit perturbations (see Auvergne et al. 2009). In our analysis, we discarded all flagged points, as well as those few points that are listed with a negative flux error.

\subsection{Spectral window}

The spectral window for the HD 46223 observations below $10 \mathrm{~d}^{-1}$ (Fig. 1) is characterised by a first peak at $f=2.007 \mathrm{~d}^{-1}$



Fig. 1. Spectral window of the HD 46223 observations. The inset zooms in on the low-frequency region.

and its double at about $f=4.011 \mathrm{~d}^{-1}$. This is related to the passage of the satellite, twice in a sidereal day, through the SAA. The relative amplitude of these two peaks is $3.5 \%$ and $1.1 \%$, respectively. In addition, owing to the orbital period of the satellite $(6184 \mathrm{~s})$ there are gaps in the data set, which generate other structures with peaks around $f=13.972 \mathrm{~d}^{-1}$ and their harmonics.

The spectral window also exhibits a peak at $f=2699.76 \mathrm{~d}^{-1}$, corresponding to the sampling regularity of about $32.003 \mathrm{~s}$. As a consequence, the pseudo-Nyquist frequency is located at about $1350 \mathrm{~d}^{-1}$. The word "pseudo" emphasizes that the sampling is not ideally regular. In addition, because of the discarded flagged data, the duty cycle of the observations is only $86.75 \%$. Because the data for all three stars were collected simultaneously with the same instrument, the spectral window properties for the other stars are very similar (see Table 1).

\subsection{Detrending}

In common with almost all CoRoT targets, there is a long-term trend visible in the data. This decreasing slope is likely caused by the CCD ageing (Auvergne et al. 2009). To remove this trend we divided the flux counts by the best-fit linear slope to the data.

The final version of the CoRoT light curve covers $T \approx$ 34.3 days. The exact start and end times, duration, number of points, and frequency resolution are listed in Table 1. We did not convert the fluxes to magnitudes.

\subsection{Frequency-by-frequency prewhitening}

The range of variations observed in the HD 46223 CoRoT light curve (Fig. 2, top) is of the order of 16000 counts, corresponding to $8 \mathrm{mmag}$. No clear pattern in the variations is visible. To better understand the variability of the CoRoT light curve, we applied a Fourier analysis based on the Lomb-Scargle method (Lomb 1976; Scargle 1982).

The amplitude spectrum (Fig. 3, top) exhibits many peaks and appears to be noisy. The highest peak is located at about $f=$ $0.75 \mathrm{~d}^{-1}$ and the second one at about $f=0.25 \mathrm{~d}^{-1}$, but no highsignificance peaks are clearly detected, nor does there appear to be systematics, such as a constant spacing between peaks. A plot 
Table 1. CoRoT data and results from the analysis for the three stars.

\begin{tabular}{|c|c|c|c|}
\hline Parameter & HD 46223 & HD 46150 & HD 46966 \\
\hline \multicolumn{4}{|l|}{ CoRoT data } \\
\hline number of raw data, $N_{\mathrm{R}}$ & 92687 & 92695 & 92688 \\
\hline number of non-flagged data, $N$ & 80403 & 80282 & 82273 \\
\hline duty cycle $\left(N / N_{\mathrm{R}}\right.$, in $\left.\%\right)$ & 86.75 & 86.61 & 88.76 \\
\hline start date non-flagged data (HJD - 2450000$)$ & 4748.488547 & 4748.485591 & 4748.488454 \\
\hline end date non-flagged data (HJD - 2450000$)$ & 4782.819650 & 4782.819657 & 4782.805898 \\
\hline duration, $T(\mathrm{~d})$ & 34.331103 & 34.334066 & 34.317444 \\
\hline freq resolution $\left(\mathrm{d}^{-1}\right)$ & 0.029128 & 0.029126 & 0.029140 \\
\hline \multicolumn{4}{|l|}{ Spectral window } \\
\hline window peak $(\%)$ at $2.0 \mathrm{~d}^{-1}$ & 3.5 & 3.8 & 3.9 \\
\hline window peak $(\%)$ at $4.0 \mathrm{~d}^{-1}$ & 1.1 & 1.0 & 1.1 \\
\hline \multicolumn{4}{|l|}{ Stopping criterion: number of frequencies } \\
\hline $\mathrm{AIC}_{\mathrm{c}}$ & 860 & 821 & 595 \\
\hline $\mathrm{BIC}$ & 491 & 444 & 277 \\
\hline HQC & 662 & 625 & 427 \\
\hline \multicolumn{4}{|l|}{ Number of red-noise significant frequencies } \\
\hline & 59 & 50 & 53 \\
\hline \multicolumn{4}{|c|}{ Number of red-noise significant frequencies present in both halves of the observing run } \\
\hline & 10 & 7 & 3 \\
\hline \multicolumn{4}{|l|}{ Red noise - fit parameters } \\
\hline$\alpha_{0}$ (counts) & $442 \pm 31$ & $430 \pm 65$ & $416 \pm 85$ \\
\hline$\tau(\mathrm{d})$ & $0.09 \pm 0.01$ & $0.08 \pm 0.02$ & $0.17 \pm 0.05$ \\
\hline$\gamma$ & $0.96 \pm 0.02$ & $0.96 \pm 0.03$ & $0.91 \pm 0.02$ \\
\hline
\end{tabular}

of the spectrum up to the pseudo-Nyquist frequency shows very little power at high frequencies. In the subsequent analysis, we therefore limited the frequency domain to $0-100 \mathrm{~d}^{-1}$.

For a more detailed analysis, we performed a traditional prewhitening. We started by subtracting the average from the fluxes. We then determined the amplitude spectrum using the Lomb-Scargle method. We selected the frequency corresponding to the highest peak in the amplitude spectrum and fitted a sine function $\left(A_{j} \sin \left(2 \pi f_{j} t_{i}+\phi_{j}\right)\right)$ to the fluxes as a function of time $\left(t_{i}\right)$. In the fitting procedure, the semi-amplitude $\left(A_{j}\right)$ and phase $\left(\phi_{j}\right)$ are free parameters, while the frequency $\left(f_{j}\right)$ was allowed to vary within a range of $\pm 0.1 / T$ from its peak value ${ }^{1}$ (where $1 / T$ is the frequency resolution and $0.1 / T$ the step size in our frequency grid). The fitted sine function was then subtracted from the data and the amplitude spectrum was re-computed from these prewhitened data. This procedure was continued until we reached the noise level in the observations. After the prewhitening, the observed variability, whatever its nature, is thus described (down to the noise level) as a sum of terms, with each term being a sine function at a particular frequency.

\subsection{Stopping criteria}

To judge when we had reached the noise level, we explored three stopping criteria that have been discussed in the literature (Hannan \& Quinn 1979; Hannan 1980; Liddle 2007). The information content of the fit is given by

$$
\begin{aligned}
c_{N}(m)= & -2 \ln \left(L_{N}(m)\right) \\
& + \begin{cases}2 m+\frac{2 m(m+1)}{(N-m-1)} & \text { Akaike, corrected }\left(\mathrm{AIC}_{\mathrm{c}}\right) \\
m \ln (N) & \text { Bayesian (BIC) } \\
2 m \ln (\ln (N)) & \text { Hannan - Quinn (HQC) }\end{cases}
\end{aligned}
$$

1 The $\pm 0.1 / T$ value turns out to be quite close to (slightly higher than) the formal errors we obtained on the frequencies (see Eqs. (3-5) and Tables 2-4). where $N$ is the number of observations, $m$ is the number of parameters and $L_{N}(m)$ is the likelihood function. To within a constant, $-2 \ln \left(L_{N}(m)\right)$ can be written as (Press et al. 1992, Sect. 15.1)

$\sum_{i=1}^{N} \frac{\left(y_{i}-F\left(t_{i}, \boldsymbol{\Theta}_{m}\right)\right)^{2}}{\sigma_{i}^{2}}$,

where $y_{i}$ are the fluxes observed at times $t_{i}$, and $\boldsymbol{\Theta}_{m}$ is the vector containing the $m$ fit-parameters. For the value of $\sigma_{i}$ we took the error bars listed with each observed flux. We stopped the prewhitening process when $c_{N}(m)$ had reached a minimum. This is very similar to least-squares minimization, but the additional terms in Eq. (1) correct for the improving of the model fit with an increasing number of parameters. We set $m=3 k$, counting three parameters (frequency, amplitude, phase) for each of the $k$ sine functions we fit. Note that we subtracted the average from the observed fluxes before starting the prewhitening procedure (Sect. 2.4), which could lead to a bias.

Applying this to the HD 46223 data, we found that the minimum of $\mathrm{AIC}_{\mathrm{c}}$ is reached at 860 terms, BIC at 491 and HQC at 662. To judge the reliability of these stopping criteria, we ran a number of simulations where we constructed artificial light curves with a given number of sine functions. We used the same time sampling as for the observations and attributed to it the same noise level and flagging (this approach is used in all simulated light curves discussed in this paper). For the amplitudes and frequencies we took either values from the analysis of the observations, or randomly drawn values distributed according to the statistics of the observed spectrum. Noise was added to the simulation using a Gaussian random variable (with mean zero and standard deviation unity) multiplied by the listed error on the observed flux. We then applied our prewhitening procedure to the simulations and compared the results of the stopping criteria with the known number of terms we introduced. It turns out that BIC gives the best value, which is always within a factor 1.5 of the correct result. Degroote et al. (2009b) also used BIC in 


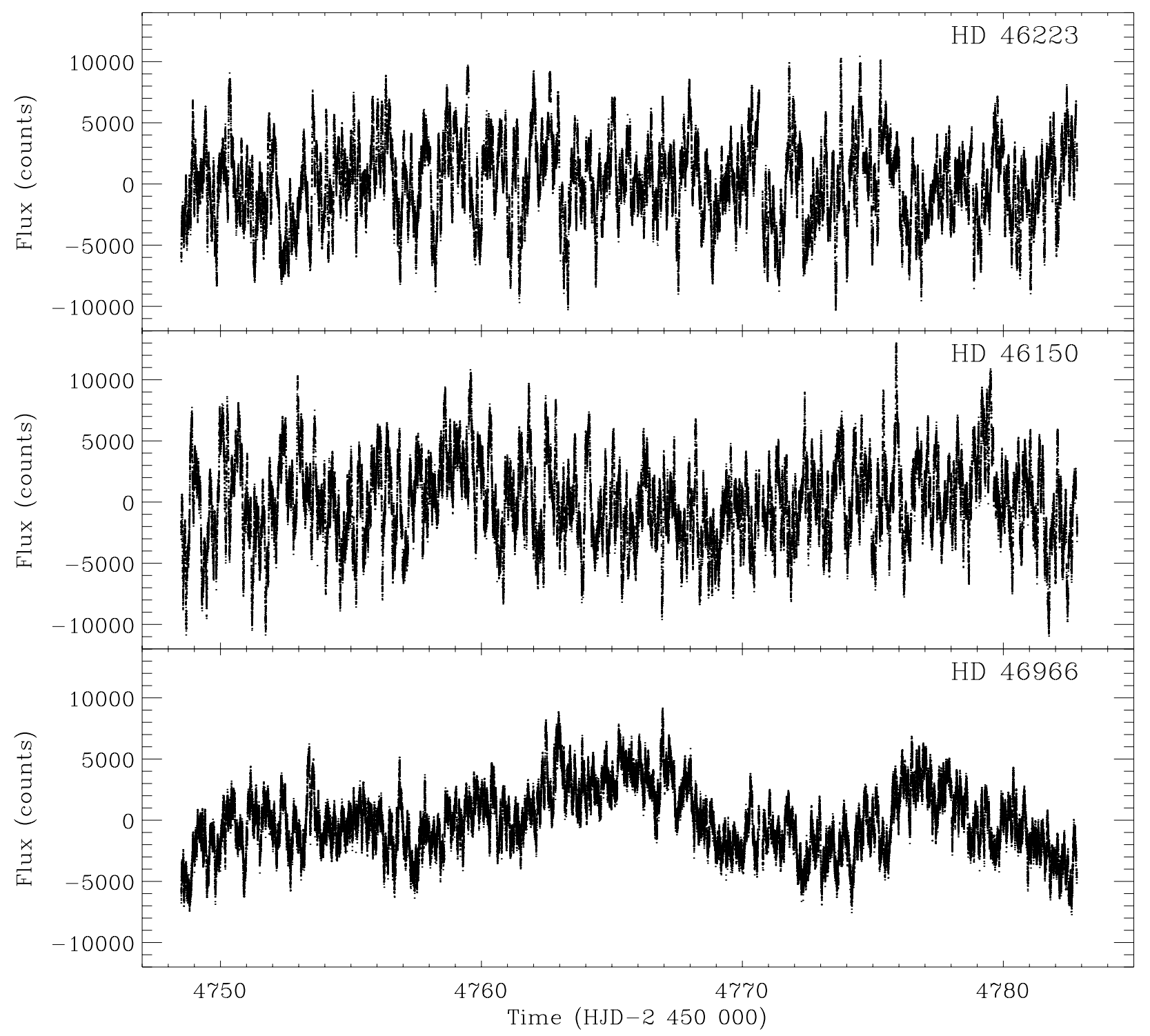

Fig. 2. Detrended CoRoT light curves, covering the full 34.3 days of the observing run. Each observation is represented by a dot. Flagged data are not plotted. Top: HD 46223, middle: HD 46150, bottom: HD 46966.

preference over the (uncorrected) AIC as a stopping criterion in their study of the $\beta$ Cep star HD 180642.

As an additional test, we also repeated the analysis on the observed data after rebinning over a number of points (up to 32 points, i.e. $17 \mathrm{~min}$ ), but found approximately the same results. This is consistent with the fact that there is little power at higher frequencies. Based on the above, we took the BIC rounded-off value of 500 terms as the stopping criterion. The final 500 frequencies and corresponding semi-amplitudes and phases are listed in Table 2. The phases are defined with respect to $t=0$ corresponding to the first non-flagged data point (as listed in Table 1).

New frequencies found during the prewhitening procedure can be quite close to previously found frequencies. The resolution criterion for this is that frequencies should be separated by at least $1.5 / T$ to be considered unique (Loumos \& Deeming 1978). Applying this to our list of 500 frequencies, we find that 276 of them are unique.
The canonical errors estimated on these frequencies $(f)$, and their corresponding semi-amplitudes $\left(A_{f}\right)$ and phases $\left(\phi_{f}\right)$ are given by the expressions of Lucy \& Sweeney (1971) and Montgomery \& O’Donoghue (1999):

$$
\begin{aligned}
\epsilon(f) & =\sqrt{\frac{6}{N}} \frac{1}{\pi T} \frac{\sigma_{f}}{A_{f}} \\
\epsilon\left(A_{f}\right) & =\sqrt{\frac{2}{N}} \sigma_{f} \\
\epsilon\left(\phi_{f}\right) & =\sqrt{\frac{2}{N}} \frac{\sigma_{f}}{A_{f}},
\end{aligned}
$$

with $\sigma_{f}$ the standard deviation on the light curve at the current stage of prewhitening. These formal errors are also listed in Table 2. 


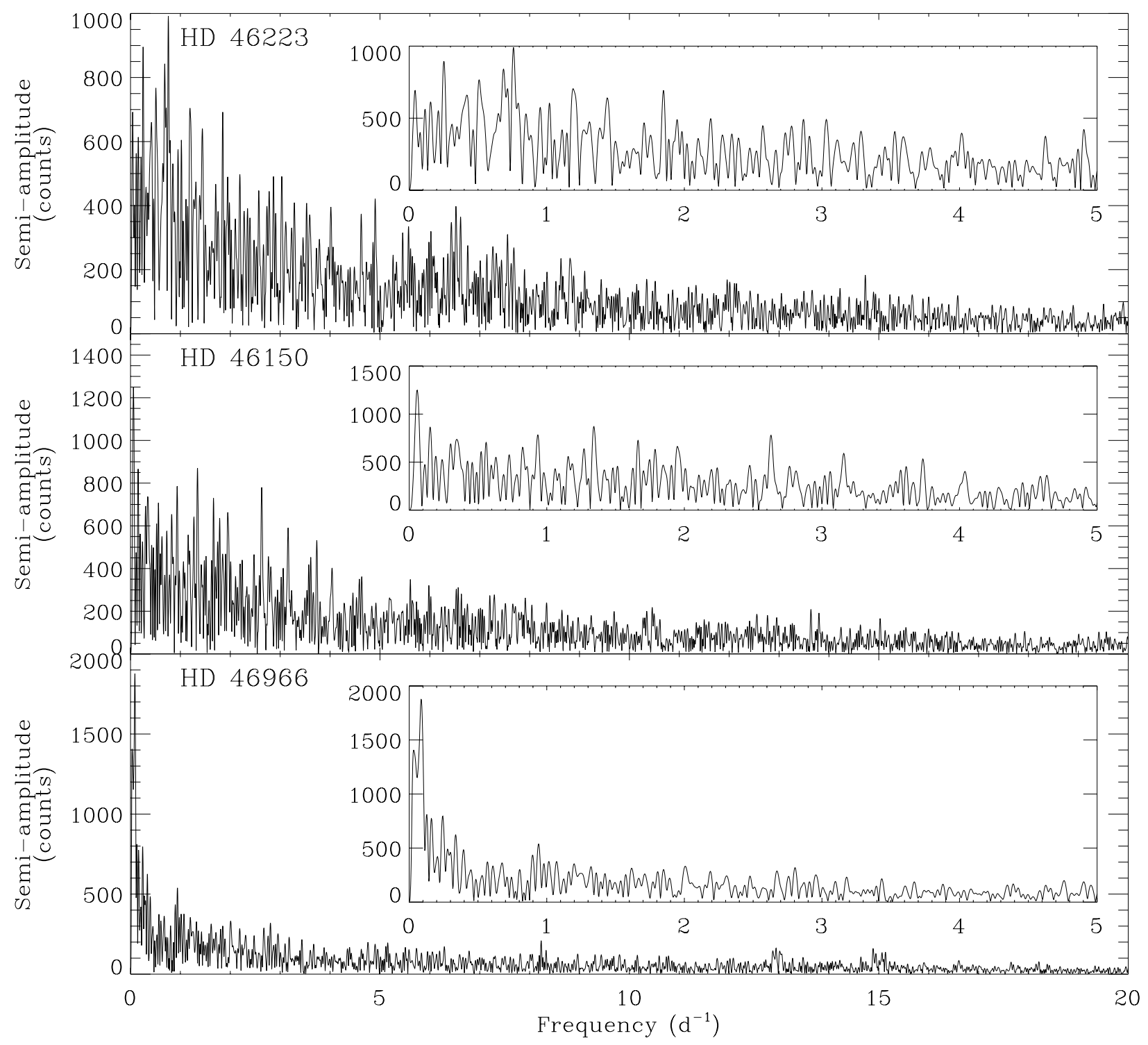

Fig. 3. Amplitude spectrum of the CoRoT light curves computed by the Lomb-Scargle method. The insets show a zoom-in of the amplitude spectrum on the low-frequencies domain. Top: HD 46223, middle: HD 46150, bottom: HD 46966.

\subsection{Multifrequency fitting}

The Lomb-Scargle-based iterative prewhitening consists in a one-by-one determination of the sine functions and in their removal from the light curve. In a gapped or unevenly sampled data set, the height of peaks depends on the height of other peaks in the periodogram. A more general procedure should therefore determine all sine function terms simultaneously.

To be more reliable in the detection of the frequencies, we also used a multifrequency algorithm introduced by Gosset et al. (2001, their Eqs. (A13) to (A19)), which was developed from the Heck et al. (1985, hereafter HMM) method. We note that this multifrequency algorithm is not based on the Lomb-Scargle technique. However, for CoRoT data we found that the amplitude spectrum computed from Lomb-Scargle and from HMM agree very well. The multifrequency algorithm takes into account the mutual influence of peaks by fitting them all together, and is therefore in principle an improvement on the one-byone prewhitening procedure used in Sect. 2.4. Similarly to the prewhitening procedure, the present method refines the value of each frequency (allowing it to vary within the natural width of the individual peaks).

We applied this method to the non-flagged data of the HD 46223 CoRoT light curves, but the great number of data points involves an excessive computation time. Accordingly, we had to deal with a limited number of terms at the same time. We found that the values of the frequencies fitted by the multifrequency programme are very similar to those determined by the prewhitening procedure and show only small differences in the amplitudes. We therefore do not list these results.

\subsection{Significance levels}

Although the quality of the CoRoT curve is unparalleled, it is still important to check whether peaks detected in the amplitude spectrum might result from a random variation rather than representing a periodic signal. To do this, we used the statistical criterion presented by Gosset (2007), which was quoted in Mahy et al. (2011). The probability that at least one of the 
Table 2. List of sine function terms for HD 46223.

\begin{tabular}{|c|c|c|c|c|c|c|c|c|c|c|}
\hline ID & $\begin{array}{l}\text { Freq. } \\
\left(\mathrm{d}^{-1}\right)\end{array}$ & $\begin{array}{l}\text { Freq. } \\
\text { Error } \\
\left(\mathrm{d}^{-1}\right)\end{array}$ & $\begin{array}{c}\text { Semi-ampl. } \\
\text { (counts) }\end{array}$ & $\begin{array}{c}\text { Semi-ampl. } \\
\text { Error } \\
\text { (counts) }\end{array}$ & $\begin{array}{l}\text { Phase } \\
\text { (rad) }\end{array}$ & $\begin{array}{l}\text { Phase } \\
\text { Error } \\
\text { (rad) }\end{array}$ & $\begin{array}{c}\text { Standard } \\
\text { Deviation } \\
\text { (counts) }\end{array}$ & $\begin{array}{l}\text { Sig. } \\
\text { Red- } \\
\text { noise }\end{array}$ & $\begin{array}{c}\text { Red- } \\
\text { noise } \\
\text { Significant }\end{array}$ & $\begin{array}{l}\text { Half- } \\
\text { run } \\
\text { Test }\end{array}$ \\
\hline 1 & 0.7548 & 0.0050 & 989.095 & 305.732 & -2.500 & 0.309 & 3296.681 & 1.0000 & & * \\
\hline 2 & 0.2493 & 0.0068 & 882.119 & 371.151 & -1.830 & 0.421 & 3221.288 & 1.0000 & & * \\
\hline 3 & 0.5102 & 0.0067 & 772.225 & 323.271 & 1.476 & 0.419 & 3160.377 & 1.0000 & & * \\
\hline 4 & 0.6858 & 0.0063 & 752.212 & 296.426 & -0.497 & 0.394 & 3112.720 & 1.0000 & & * \\
\hline 5 & 1.1944 & 0.0056 & 699.589 & 244.181 & 0.676 & 0.349 & 3067.088 & 1.0000 & & * \\
\hline 6 & 1.8456 & 0.0046 & 691.462 & 199.852 & -2.537 & 0.289 & 3026.889 & 1.0000 & & * \\
\hline 7 & 0.0395 & 0.0091 & 681.390 & 385.046 & -1.697 & 0.565 & 2986.975 & 1.0000 & & * \\
\hline 8 & 0.4251 & 0.0077 & 650.217 & 312.906 & -0.054 & 0.481 & 2948.151 & 1.0000 & & * \\
\hline 9 & 0.9477 & 0.0064 & 636.137 & 251.785 & -1.726 & 0.396 & 2912.468 & 1.0000 & & $*$ \\
\hline 10 & 1.4370 & 0.0053 & 642.207 & 212.699 & -1.822 & 0.331 & 2877.663 & 1.0000 & & $*$ \\
\hline$\cdots$ & $\cdots$ & $\cdots$ & $\cdots$ & $\cdots$ & $\cdots$ & $\cdots$ & $\cdots$ & $\cdots$ & $\cdots$ & $\cdots$ \\
\hline 59 & 7.5519 & 0.0027 & 303.826 & 51.957 & 0.500 & 0.171 & 1913.541 & 0.0080 & $\mathrm{R}$ & \\
\hline$\ldots$ & $\ldots$ & $\ldots$ & $\ldots$ & $\ldots$ & $\ldots$ & $\ldots$ & $\ldots$ & $\ldots$ & $\ldots$ & $\ldots$ \\
\hline
\end{tabular}

Notes. This is an excerpt of Table 2. The full table is available in electronic form at the CDS. We list the first ten terms as well as the first term that is significant under the assumption of red noise (indicated with "R"). Terms that are found in both halves of the observing run are indicated with “*” in the last column.

semi-amplitudes of an extended set of frequencies exceeds a threshold $z$ under the null hypothesis of a stochastic process of variance $\sigma_{f}^{2}$ is given by

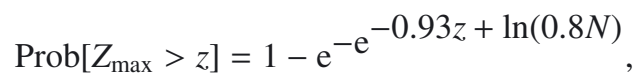

where $Z_{\max }=\max _{f<f_{\mathrm{Ny}}} Z(f), N$ is the number of points in the data set and $Z(f)=A_{f}^{2} N /\left(4 \sigma_{f}^{2}\right)$ with $A_{f}$ the semi-amplitude of the frequency $f$ and $\sigma_{f}$ the standard deviation on the light curve at the current stage of prewhitening.

We also explored a different version of this criterion, which uses (Degroote et al. 2009a)

$\operatorname{Prob}\left[Z_{\max }>z\right]=1-(1-\exp (-z))^{N_{i}}$,

where $N_{i}$ is the number of independent frequencies. Degroote et al. show that the CoRoT data are sufficiently well equallyspaced that we can take $N_{i}=2 N$. In our work, the first criterion (given by Eq. (6)) turns out to be slightly stricter, and we therefore discuss only results obtained with that one.

In applying this criterion, the simplest approach is to assume white noise, i.e. $\sigma_{f}$ is independent of frequency and its value is given by the standard deviation on the light curve at the current stage of prewhitening. Under that assumption all 500 sine function terms are highly significant.

\subsection{Red noise}

It is quite clear from the amplitude spectrum (Fig. 3) that the power increases substantially towards lower frequencies. This strongly suggests the presence of red noise. We stress that the word "noise" does not imply an instrumental origin but rather points to predominantly stochastic behaviour: in Sect. 6.2 we discuss the possible physical cause of the red noise.

In the present section, we determine the observed properties of this red noise. Stanishev et al. (2002) suggest to describe the amplitude spectrum $\alpha(f)$ by using the following fit-function:

$\alpha(f)=\frac{\alpha_{0}}{1+(2 \pi \tau f)^{\gamma}}$,

with $\alpha_{0}$ the scaling factor, $\gamma$ the slope of the linear part (in a $\log -\log$ plot) and $\tau$ an estimation of the mean duration of the dominant structures in the light curve. We fitted this profile to the amplitude spectrum of HD 46223 (Fig. 4, top) to quantify the information about the red noise contained in the periodogram. We applied a least-squares fit to the logarithm of both frequency and semi-amplitude, limiting the frequencies used to $0-100 \mathrm{~d}^{-1}$. The resulting fit parameters and their 1-sigma error bars are listed in Table 1 .

To re-evaluate the significance of the peaks under the hypothesis of red noise, we need to determine its $\sigma_{f \text {,red }}$. We did this by first converting the fit-function for amplitude (Eq. (8)) into power. We then re-scaled that function so that its integral over frequency (up to the pseudo-Nyquist frequency of $\sim 1350 \mathrm{~d}^{-1}$ ) is the same as the variance on the flux multiplied by the pseudoNyquist frequency.

We then repeated the prewhitening procedure and determined for each frequency the significance of the peak by using Eq. (6) with $Z(f)=A_{f}^{2} N /\left(4 \sigma_{f \text {,red }}^{2}\right)$. It is important to realise that in doing so, we still select the highest peak at each step in the prewhitening process, not the one that is most significant under red-noise conditions. If we were to select the most significant peak, it would correspond to a small-amplitude contribution to the light curve. In the prewhitening procedure (Sect. 2.4) we would then need to fit a sine function with this frequency to the light curve, and subtract it. The fitting procedure will not succeed, because in attempting to fit only a minor contribution to the light curve, it will be led astray by the much higher-amplitude contributions (which have not been removed yet). It will therefore give an incorrect result, or will fail to converge.

Applying this procedure, we found 59 significant sine functions with a significance level below 0.01 ; we indicate them with an "R" in Table 2.

\subsection{Split into two halves}

We next applied an additional method to determine the reliability of the sine function terms we found. We split the data set into two halves, and repeated the analysis separately on each half. We then checked how many of the original terms can also be found in both halves. We considered two frequencies $\left(f_{1}\right.$ and $\left.f_{2}\right)$ to be the same if $\left|f_{1}-f_{2}\right|<\sqrt{\epsilon^{2}\left(f_{1}\right)+\epsilon^{2}\left(f_{2}\right)}$, where the errors are defined in Eq. (3). Simulations with artificial data confirm that 



Fig. 4. Fit-function (Eq. 8, red line) to the amplitude spectra as a function of frequency, in a log-log plot. Top: HD 46223, middle: HD 46150, bottom: HD 46966.

this is the appropriate criterion to use. We could of course relax it by considering equality to within 2 or 3 times the 1-sigma error bar, but this would considerably increase the number of false coincidences.

If we first look at the terms determined assuming white noise, we find that of the 500 terms extracted from the full run, 202 are present in both halves. This is substantially less than the 282 terms we find if we construct an artificial, noisy, light curve from the first 100 observed terms of HD 46223. Other simulations with frequencies drawn randomly according to the observed distribution also find a significantly larger number of terms in both halves.

If we limit ourselves to the 59 red-noise significant terms listed in Table 2, we find that only 10 are present in both halves of the data set.

\subsection{Frequency combinations and spacings}

We next searched for the presence of low-order harmonics (up to order 10) in the list of 500 frequencies. With the usual criterion that frequencies should be equal to within the square root of the sum of the squares of the individual 1-sigma errors, we found 983 such harmonics. However, simulations as in Sect. 2.5 show
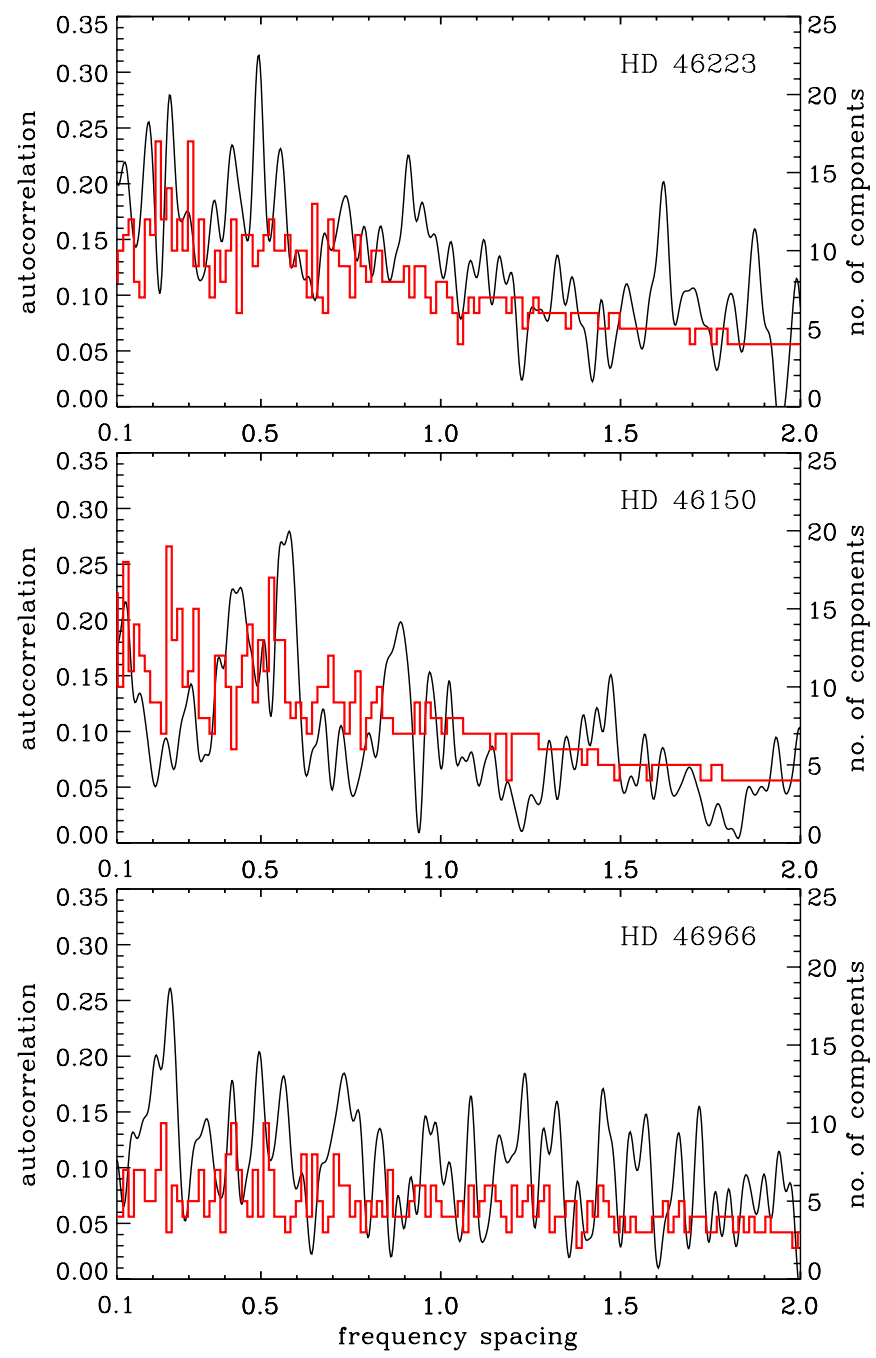

Fig. 5. Autocorrelation functions (black curve) of the power periodograms and number of components in a chain with a given frequency spacing (red curve). Top: HD 46223, middle: HD 46150, bottom: HD 46966.

a factor 2-3 more harmonics. Our findings must therefore be attributed to coincidences, not to true harmonics.

A similar search for linear combinations of the type $f_{1}+f_{2}=$ $f_{3}$ among the 500 frequencies revealed 45412 results. But, again, simulations showed these to be spurious. If we limit our search to frequencies significant assuming the red-noise hypothesis, we find no harmonics and only one linear combination. The reason we found so few of these is that most of our red-noise frequencies are between 10 and $20 \mathrm{~d}^{-1}$; harmonics and sums of these are then outside the $10-20 \mathrm{~d}^{-1}$ range. Because of the limited value of these results, we do not list them.

In addition to these linear combinations, we investigated the frequency spacing for HD 46223. Degroote et al. (2010) found such spacing for the O8 V star HD 46149. Following their work, we looked at both the autocorrelation function of the power periodogram and the number of components we find in a chain $\left(f_{0}\right.$, $\left.f_{0}+\Delta f, f_{0}+2 \Delta f, \ldots\right)$ with an assumed frequency spacing $\Delta f$. In both the chain and autocorrelation test, we started from the 500 frequencies, but eliminated those outside the 3.0-12.0 $\mathrm{d}^{-1}$ domain. The lower limit is taken to avoid the stronger peaks, the upper limit to avoid effects from the satellite orbital period $\left(13.972 \mathrm{~d}^{-1}\right)$. To determine the number of components in a chain 

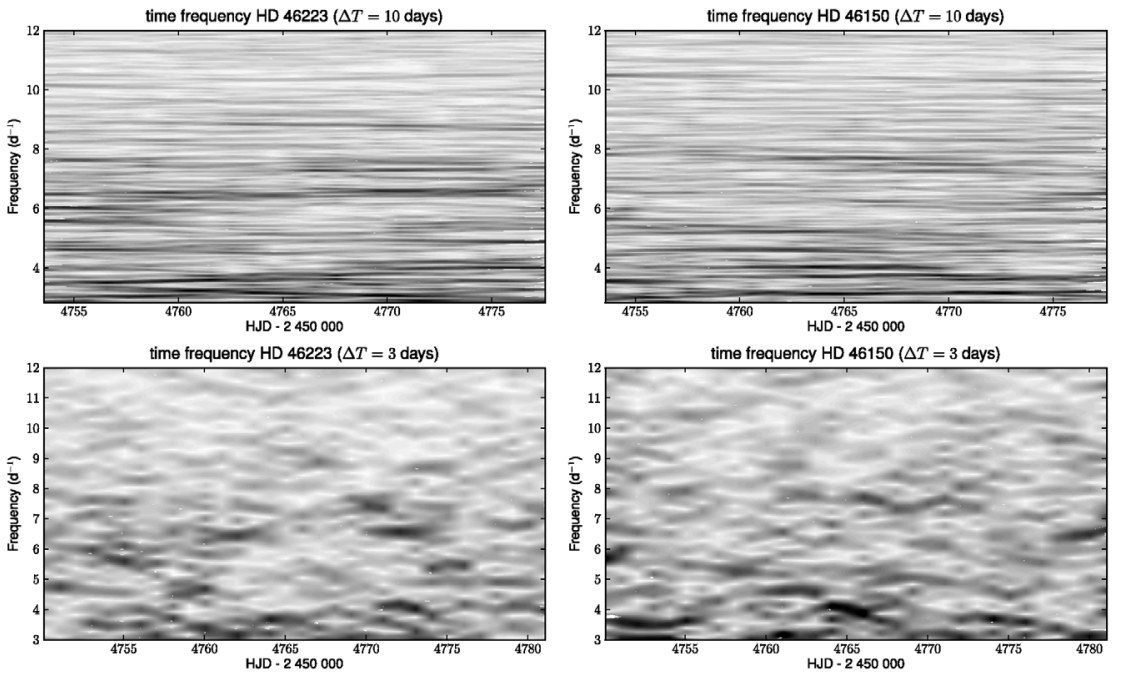

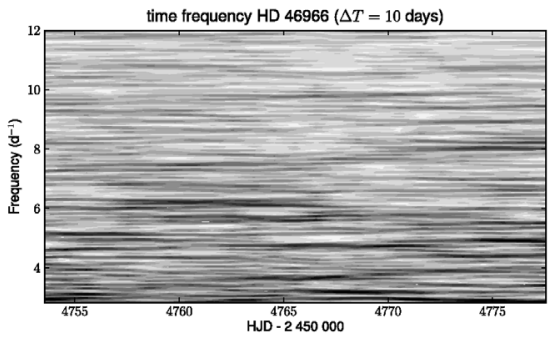

time frequency HD $46966(\Delta T=3$ days $)$

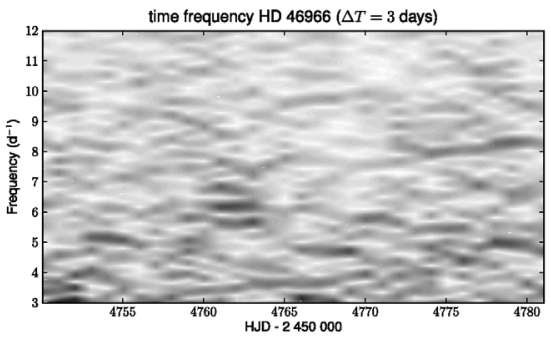

Fig. 6. Time-frequency analysis of the light curves. The top row uses a sliding window of 10 days, the bottom row one of 3 days. Left panels: HD 46223, middle: HD 46150, right: HD 46966. The grey scale indicates the semi-amplitude of the frequencies. Clearly the frequencies do not have long lifetimes.

at a given frequency, we considered that $f_{0}+n \Delta f$ equals a listed frequency if both differ by less than the step size we used in the figure, which is $0.015 \mathrm{~d}^{-1}$. For $\Delta f$, we considered only the range of 0.1 to $2.0 \mathrm{~d}^{-1}$, because this is where both the autocorrelation and chain-test function have their highest values.

Figure 5 (top) shows the results for HD 46223. The autocorrelation function has a peak at $\Delta f=0.5 \mathrm{~d}^{-1}$, but the number of components does not confirm this peak. We therefore conclude that we do not detect significant frequency spacings in HD 46223.

\subsection{Time-frequency analysis}

We also performed a time-frequency analysis (Fig. 6) to detect variations in the amplitude of frequencies or in the frequencies themselves as a function of time.

For this purpose, a sliding window with lengths of 10 and 3 days was applied to the light curve and shifted with a step of one day. Then we applied a Fourier analysis on each frame by using the HMM method (for further details on this time-frequency analysis method, see Huat et al. 2009). A plot of all frequencies in the $3-12 \mathrm{~d}^{-1}$ range detected on a sliding window of 10 and 3 days is shown in Fig. 6. This figure confirms what we already found from the test where we split the data into two halves. The frequencies are clearly not stable over the $\sim 34$-day timescale of the observation. The figures do not show many frequencies that have a lifetime longer than the duration of the sliding window. This behaviour is compatible with the dominant presence of red noise.

The limited lifetime also raises doubts about the applicability of the $1.5 / T$ criterion (Loumos \& Deeming 1978) to resolve frequencies (see Sect. 2.5). A limited lifetime translates into a broadening of the frequency. This will reduce the number of unique frequencies found in our analysis.

\section{HD 46150}

For the analysis of HD 46150 we applied a similar detrending as for HD 46223. The range of variations observed in the HD 46150 CoRoT light curve (Fig. 2, middle) is of the order of 16000 counts, corresponding to $8 \mathrm{mmag}$. More details are listed in Table 1. No clear pattern in the variations is visible. The amplitude spectrum (Fig. 3, middle) is noisy and has its highest peak at $0.055 \mathrm{~d}^{-1}$. Because there is little power at high frequencies, we limited the frequency domain to $0-100 \mathrm{~d}^{-1}$ in the subsequent analysis.

We next applied our standard prewhitening procedure and checked the stopping criteria (Eq. (1)). From the values found (Table 1) we decided to limit the number of terms to 500 . This list of frequencies and corresponding semi-amplitudes and phases is given in Table 3 (this table is only available in electronic form at CDS). Applying the significance criterion (Eq. (6)) with the assumption of white noise results in all 500 terms being significant. The Loumos \& Deeming (1978) test shows 296 of these to be unique. Multifrequency fitting (see Sect. 2.6) gives basically the same set of terms, though with slightly different amplitudes.

However, it is again clear that there is a substantial red-noise component present in the spectrum, which we fit with the fitfunction of Eq. (8) (see Table 1 and Fig. 4, middle). Judging the significance of peaks with respect to this red noise, we found 50 significant terms (indicated with " $R$ " in Table 3), using a 0.01 cut-off.

When we split the observed data set into two, we found that 195 of the 500 terms occur in both halves, but this is less than the simulations of Sect. 2.9 show. Under the red-noise hypothesis, only seven of the 50 terms are present in both halves. We also explored the linear combinations of all 500 frequencies, but simulations show these to be not significant, as for HD 46223 (see Sect. 2.10). We found no linear combinations among the rednoise frequencies unless we relaxed the criterion we had implemented for considering two frequencies to be equal. This shows that there are no significant linear combinations.

We next applied a similar frequency spacing test as for HD 46223. A peak in the autocorrelation function was found at $\Delta f=0.58 \mathrm{~d}^{-1}$, but it was not confirmed by the plot with the number of components in a chain (Fig. 5, middle). Finally, a time-frequency analysis (Fig. 6, middle) showed short "lifetimes" for the frequencies, which is again consistent with red noise. 

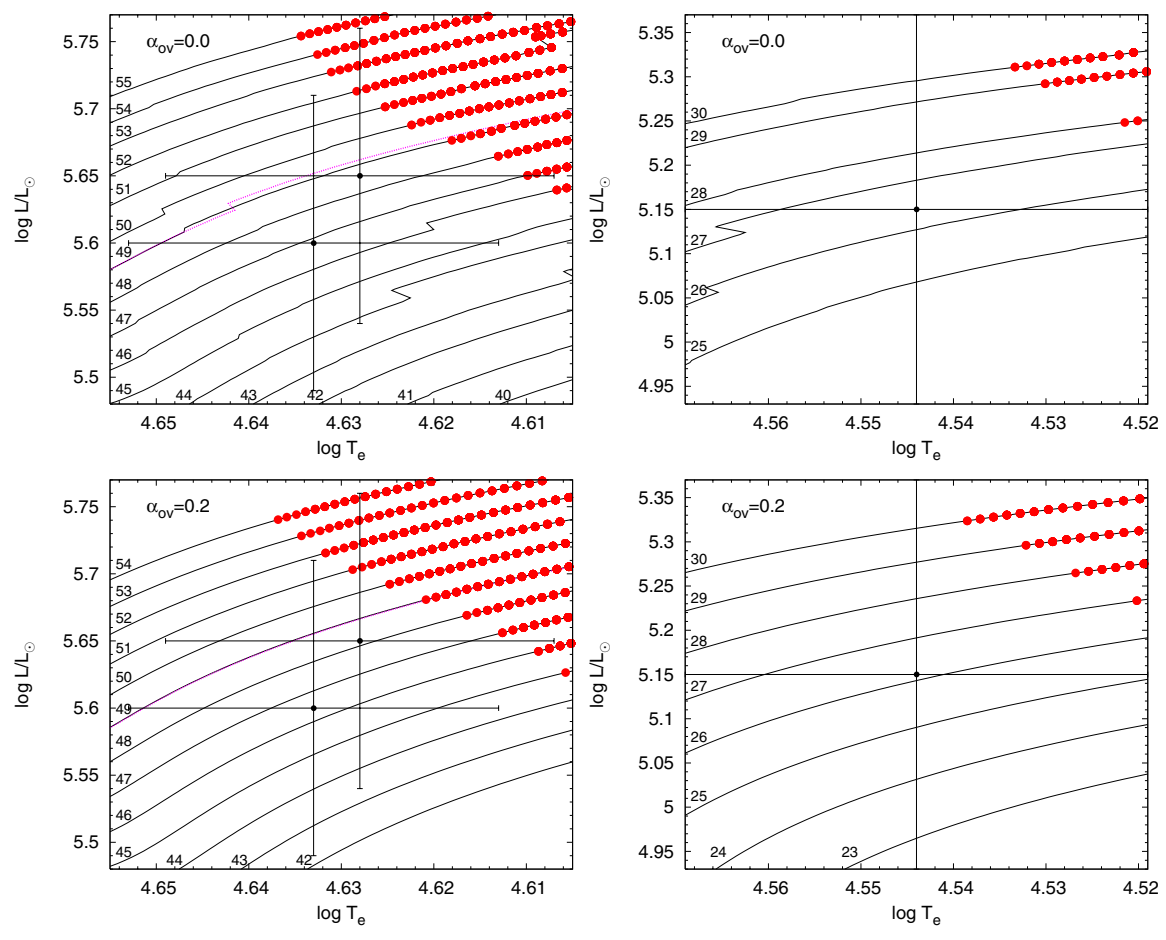

Fig. 7. Evolutionary tracks relevant for our three stars. The models in the bottom figures include overshooting, the ones at the top do not. Left: tracks of 40 to $55 M_{\odot}$ computed with a mass-loss rate of $6.0 \times 10^{-8} M_{\odot} \mathrm{yr}^{-1}$. The error boxes of HD 46223 (the hotter star) and HD 46150 (the cooler star) are also displayed. To show that both stars can be modelled by the same mass-loss rate without affecting the excited frequency domain, we also plot a $49 M_{\odot}$ track computed with $\dot{M}=6.8 \times 10^{-8} M_{\odot} \mathrm{yr}^{-1}$ (magenta-coloured line). Right: same, but for HD 46966 computed with a mass-loss rate of $5.0 \times 10^{-9} M_{\odot} \mathrm{yr}^{-1}$. The full circles indicate positions along the tracks that have excited frequencies.

\section{HD 46966}

The treatment of the data of HD 46966 was similar to that of HD 46223 and HD 46150 and details are listed in Table 1. The CoRoT curve of HD 46966 (Fig. 2, bottom) exhibits an almost regular oscillation with an approximate period of 10 days, but only about three cycles are covered by the observations. In addition, we detected variability on shorter timescales. The peak-topeak variation amplitudes correspond to about 6 mmag.

Because there is little power at high frequencies, we limited the frequency domain to $0-100 \mathrm{~d}^{-1}$. We applied our standard prewhitening procedure and checked the stopping criteria (Eq. (1)). From the values found (Table 1), we decided to limit the number of terms to 300 . The list of frequencies and corresponding semi-amplitudes and phases is given in Table 4 (this table is only available in electronic form at CDS). The Loumos \& Deeming (1978) criterion shows 196 of these to be unique. The amplitude spectrum (Fig. 3, bottom) is characterised by a first peak that is three times larger than the second one. This peak, at $f=0.084 \mathrm{~d}^{-1}$, i.e. 12 days, corresponds to the observed almost regular oscillation.

It is, however, better to consider red noise, described by the fit-function from Eq. (8). Applying this, we found 53 significant terms, with a cut-off of 0.01 . They are indicated with an " $R$ " in Table 4. Note that in this analysis the $f=0.084 \mathrm{~d}^{-1}$ frequency discussed above is no longer considered significant. Table 4 shows that it has a false-alarm probability of 0.997 , which is too high compared to our 0.01 cut-off. Multifrequency fitting (see Sect. 2.6) gives basically the same set of frequencies, though with slightly different amplitudes.

The test where we split the observed data set into two showed that only 77 of the 300 terms were found in both halves. For the terms that are significant under the red-noise hypothesis, only three were found in both halves. Simulations as in Sect. 2.9 show that these numbers are not significant.

We also explored the linear combinations between the 300 frequencies, but simulations show that these are not significant, as for the two other stars. Limiting ourselves to the
53 red-noise frequencies, we found no linear combinations. Only when we increase the criterion that we employ for equality of frequencies by a factor of three did we begin to find linear combinations. The result is therefore not significant.

We also applied a similar frequency spacing test as for HD 46223. Again, a peak in the autocorrelation function was found, at $\Delta f=0.25 \mathrm{~d}^{-1}$. The plot with the number of frequencies in a chain peaks at a different value of $\Delta f=0.43 \mathrm{~d}^{-1}$, however (Fig. 5, bottom), showing the result to be not significant. As for the two other stars, the time-frequency analysis (Fig. 6, bottom) shows short lifetimes for the frequencies; this again indicates the presence of red noise.

\section{Theoretical pulsations}

An important goal of the CoRoT photometry is to detect radial or non-radial pulsation modes in massive stars. To predict which modes can be expected, we computed a grid of models by using the Rome stellar evolution code ATON (Ventura et al. 2008). In these calculations, we assumed a metallicity of $Z=0.015$ and the metal mixture of Grevesse \& Noels (1993). We also used the MAD non-adiabatic pulsation code (Dupret et al. 2003) to determine the frequencies of excited modes. The computations were made on the basis of preliminary stellar and wind parameters derived from the atmosphere code CMFGEN (Hillier \& Miller 1998) by Martins et al. (in prep.). These parameters are listed in Table 5. We note, however, that we used the same value for the mass-loss rate for HD 46223 as for HD 46150 to compute the evolutionary tracks. The difference between the value used and those listed in Table 5 does not significantly affect our results. Additionally, we considered models both with and without overshooting.

HD 46223 and HD 46150 are in very similar positions in the HR diagram, whilst HD 46966 is cooler and more evolved. All three stars lie just at the boundary of the instability strip of the $\kappa$-mechanism. Theoretical excited frequencies for that mechanism are generally in the range of $2-5 \mathrm{~d}^{-1}$ for $l=0$ to $l=4$ and 
Table 5. Stellar parameters for the three stars.

\begin{tabular}{lccc}
\hline \hline Parameter & HD 46223 & HD 46150 & HD 46966 \\
\hline $\log T_{\text {eff }}(\mathrm{K})$ & $4.633 \pm 0.020$ & $4.628 \pm 0.021$ & $4.544 \pm 0.025$ \\
$\log g(\mathrm{cgs})$ & 4.00 & 4.00 & 3.75 \\
$\log L / L_{\odot}$ & $5.60 \pm 0.11$ & $5.65 \pm 0.11$ & $5.15 \pm 0.22$ \\
$R / R_{\odot}$ & 11.39 & 12.35 & 10.24 \\
$\log M\left(M_{\odot} \mathrm{yr}^{-1}\right)$ & -7.20 & -7.17 & -8.30 \\
$v_{\text {eq }} \sin i\left(\mathrm{~km} \mathrm{~s}^{-1}\right)$ & 100 & 100 & 50 \\
$v_{\text {macro }}\left(\mathrm{km} \mathrm{s}^{-1}\right)$ & 32 & 37 & 27 \\
\hline
\end{tabular}

Notes. Preliminary stellar and wind parameters derived by Martins et al. (in prep.).

a non-radial mode is also excited around $12 \mathrm{~d}^{-1}$. The theoretical frequencies from our model calculations are shown in Fig. 8 for 30 and $52 M_{\odot}$ (without overshooting): models with these masses present the largest number of excited modes. Models computed with overshooting give similar results.

For HD 46223 we considered stellar evolution models with an initial mass ranging from 45 to $53 M_{\odot}$ (Fig. 7, left panel). On these tracks, models with a mass greater than $47 M_{\odot}$ show excited modes. For this mass range, the excited modes all have frequencies around $4 \mathrm{~d}^{-1}$. However, if we look at the observed amplitude spectrum of HD 46223 (Fig. 3, top), no outstanding peak is situated close to $4 \mathrm{~d}^{-1}$, which means that this star probably does not present pulsations, neither radial nor non-radial. For HD 46150 a similar conclusion can be drawn. There are lowamplitude peaks around $4 \mathrm{~d}^{-1}$, but the peaks are not strong and they could be the result of noise.

The amplitude spectrum of HD 46966 is clearly dominated by peaks at the frequencies $f=0.084$ and $0.039 \mathrm{~d}^{-1}$. The time coverage of the CoRoT observations is too short to know if these are regular variations. Furthermore, it is unlikely that these two frequencies are caused by excited modes (Fig. 8, bottom) because those modes are predicted to be between 2.5 and $4 \mathrm{~d}^{-1}$. Even though the amplitude spectrum has peaks situated in this frequency region, these peaks are weak.

It is important to emphasize that the results presented in this section depend on the stellar parameters determined by Martins et al. (in prep.). These parameters are still under investigation and should therefore be considered as preliminary. Nonetheless, the present work shows that even with the unprecedented quality of the CoRoT light curves, we do not convincingly detect the predicted excited modes. This suggests that they are only weakly excited, or perhaps, not at all. On the other hand, a number of stronger peaks are observed, but at frequencies that are so different from the theoretical ones that we cannot attribute them to the expected excited modes, at least not within the framework of current theoretical models. It is thus not possible to conclude with certainty that these three stars are pulsating. Moreover, we also explored the possibility of solar-like oscillations because Degroote et al. (2010) found such oscillations in the OB binary system HD 46149. But the existence of these oscillations was rejected in HD 46150, HD 46223, and HD 46966 because no regular spacings were found in the amplitude spectra.

\section{Discussion}

\subsection{Radius and rotational frequency}

In a presumably single star, large-scale photometric variations can have a frequency compatible with the rotational frequency
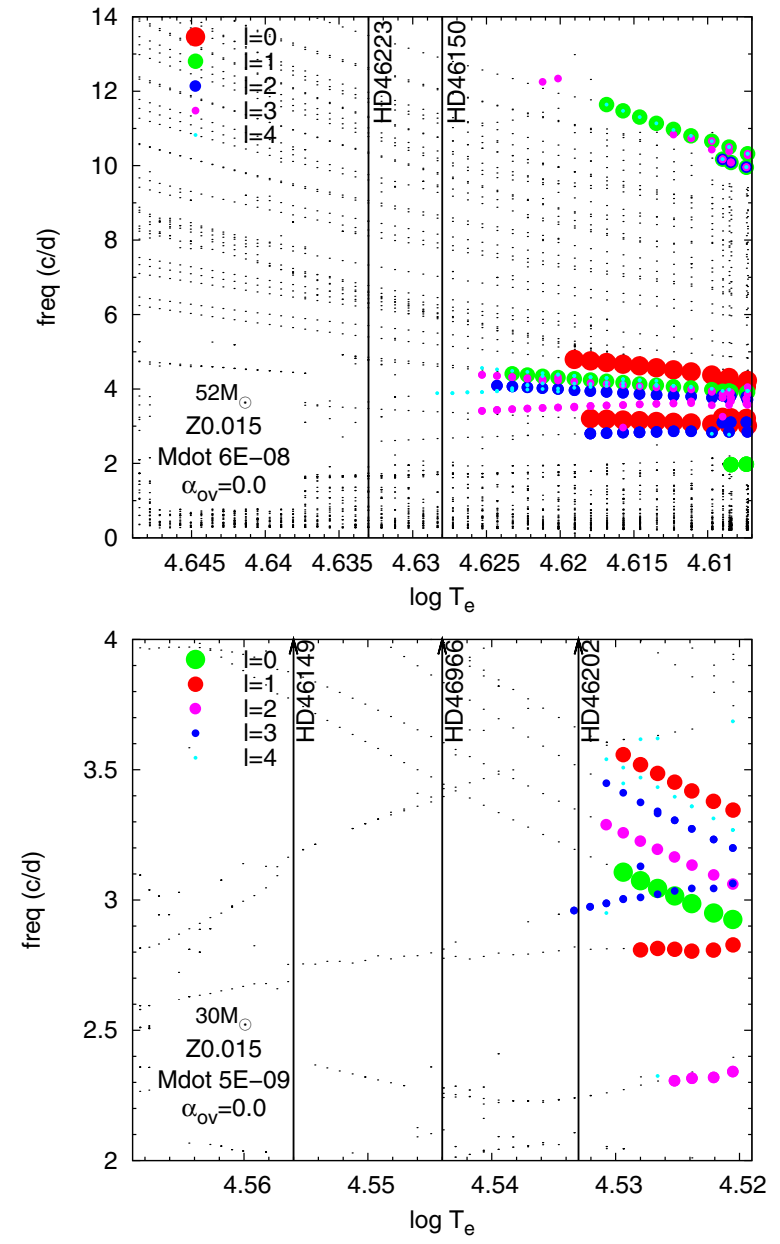

Fig. 8. Theoretical oscillation frequencies (for the $\kappa$-mechanism) computed with the MAD non-adiabatic code, as a function of the effective temperature. The results shown here do not include overshooting. Top: $52 M_{\odot}$ track. Bottom: $30 M_{\odot}$ track. The black points indicate models that do not show pulsations, the coloured full circles stand for those models with the excited frequencies. The $l$-value is colour-coded (see legend). We also indicate with vertical lines the effective temperatures of the three stars studied here, as well as HD 46149 (Degroote et al. 2010) and HD 46202 (Briquet et al. 2011).

of the star. Their exact origin could be attributed to the existence of a spot on the star or be associated with the stellar wind.

The first low frequency detected in the amplitude spectrum of HD 46223 is $f=0.249 \mathrm{~d}^{-1}$, corresponding to 4.02 days. With this period and the parameters from Table 5, we derived an equatorial velocity $v_{\mathrm{eq}}$ of about $140 \mathrm{~km} \mathrm{~s}^{-1}$, which is higher than the $v_{\text {eq }} \sin i$ value of $100 \mathrm{~km} \mathrm{~s}^{-1}$ measured by Mahy et al. (2009) and Martins et al. (in prep.). Consequently, the frequency $f=0.249 \mathrm{~d}^{-1}$ could be associated with the rotational cycle of the star, implying a rotational period of about $\sim 4$ days and an inclination of $43-45^{\circ}$.

For HD 46150 the amplitude spectrum exhibits a strong peak at $f=0.055 \mathrm{~d}^{-1}$, corresponding to 18 days. The derived equatorial velocity is $34 \mathrm{~km} \mathrm{~s}^{-1}$, which is too low compared to the $v_{\text {eq }} \sin i$. The frequency corresponding to the equatorial velocity found by Mahy et al. (2009) or Martins et al., should be close to or higher than $0.16 \mathrm{~d}^{-1}$. The list of frequencies (Table 3 ) shows that the third frequency $\left(f=0.144 \mathrm{~d}^{-1}\right)$ in Table 3 is quite close. It corresponds to an equatorial velocity of $90 \mathrm{~km} \mathrm{~s}^{-1}$, which is 
within acceptable range of the Mahy et al. and Martins et al. value, but would require an inclination of $90^{\circ}$.

The amplitude spectrum of HD 46966 is marked by a strong peak at $f=0.084 \mathrm{~d}^{-1}$, representing a period of 12 days. With the stellar parameters mentioned in Table 5, we estimate the equatorial velocity to be close to $44 \mathrm{~km} \mathrm{~s}^{-1}$. This velocity is just below the 1-sigma error bar on the $v_{\text {eq }} \sin i$ determined by Mahy et al. (2009). Martins et al. (in prep.), on the other hand, clearly show that the $v_{\text {eq }} \sin i$ is lower than $50 \mathrm{~km} \mathrm{~s}^{-1}$. Consequently, the frequency $f=0.084 \mathrm{~d}^{-1}$ could be associated with the rotational cycle of the star, implying a rotational period of about 12 days.

\subsection{Pulsations and red noise}

For the three O-type stars studied here, a substantial number of sine function terms are found. However, it is unclear how many of those - if any - can be classified as true independent modes. They do not show the linear combinations or frequency spacings that may be expected in pulsators. If these terms were interpreted as pulsational modes, then their lifetimes would be very short. The amplitude spectra for these three stars are therefore unlike any of those of known pulsators in that part of the HR diagram (Aerts et al. 2010, Chap. 2).

This is clearly illustrated by comparing the periodogram of these hot O stars (spectral types O4-O8) to that of the cooler star HD 46202 (O9 V). This star behaves like a classical pulsator, with clear $\beta$ Cep-like pulsations that dominate the power spectrum (Briquet et al. 2011). Various causes for what we see in our periodograms can be eliminated: rotational effects on pulsation can be excluded because our periodograms are very different from those of Be stars (e.g. Diago et al. 2009). Spots on the surface of the star would give clearly isolated frequencies (and linear combinations between them). A power excess due to stochastic modes would occur at higher frequencies.

The interpretation that ascribes most of the power in the periodogram to red noise therefore seems more appropriate. We stress again that this red noise is caused by a physical mechanism in the star. It is not an instrumental effect, because its behaviour is different from star to star in these simultaneous observations. The parameters of the fit-function (Table 1) show that HD 46966 has a somewhat lower $\alpha_{0}$ and a somewhat longer $\tau$ than the other two stars. Compared with Plaskett's Star (HD 47129) a clearer difference is seen: especially the $\gamma$ value is different $(\gamma=2.3$, Mahy et al. 2011). We also fitted the red-noise component of HD 46149 (Degroote et al. 2010). This star has a $\tau=29.5 \pm 12$ and $\gamma=0.55 \pm 0.01$, which are substantially different from the stars studied in this paper. All this points to an origin for the red noise that is intrinsic to the star.

To see how convincing the red-noise description is, we simulated light curves containing only red noise (according to the fit-function - Eq. (8)), and analysed them in the same way as the real data. We followed the prescription by Timmer \& Koenig (1995) to generate the red noise. In the simulated data, the number of significant red-noise frequencies found is somewhat higher (by a factor less than two) than in the real data. Only one or two of these significant red-noise frequencies can be found in both halves of the light curve (somewhat less than for the real data). The number of linear combinations found is higher than for the real data, but this is presumably related to the higher number of significant red-noise frequencies. It therefore seems that the observed data can largely be described as red noise, though a few pulsational frequencies might be present.

The presence of red noise in the stellar signal indicates stochastic, chaotic, or quasiperiodic effects. It occurs in a number of astrophysical contexts, such as the light curves of Mira variables (Templeton \& Karovska 2009) and red supergiants (Kiss et al. 2006), and the X-ray light curves of active galaxies (Vaughan et al. 2003), dwarf novae (Hakala et al. 2004) and high-mass X-ray binaries (Burderi 1994).

The physical cause of this red noise in early-type stars is not clear. Below we discuss three possibilities: sub-surface convection, granulation, and inhomogeneities in the stellar wind. We stress that all three are highly speculative and that as yet no modelling exists to see if they would give the correct quantitative behaviour.

A link between red noise and convection was already pointed out by Schwarzschild (1975). For early-type stars, Cantiello et al. (2009) show the existence of a sub-surface convection zone caused by the opacity peak due to iron ionization. The authors show that stars in the HR diagram where this zone is present also show microturbulence, non-radial pulsations, wind clumping, and line-profile variability, suggesting that these are caused by the convection zone. We speculate that the red noise detected in the early-type stars studied here is also a consequence of this convection zone, and could be associated with any of the above observational indicators. The position of our stars in the HR diagram shows that they should have a fairly weak convection zone (see Cantiello et al. 2009, their Fig. 9). This suggests that the high quality of the CoRoT data allows us to see a more subtle effect than Cantiello et al. considered. Instead of attributing the red noise to convection only, there could also be an interaction between pulsation and convection. Perdang (2009) shows that this can result in red-noise dominated light curves.

We also explored if the physical cause of the red noise could be described as granulation. Kallinger \& Matthews (2010) show that in the two $\delta$ Scuti stars they study, the many hundreds of peaks in the amplitude spectrum can also be interpreted as being caused by granulation. Their Fig. 3 relates the typical frequency of granulation (i.e. the inverse of the timescale) to a specific combination of mass, radius, and effective temperature. Although this relation is based on cooler stars, we nevertheless used it to predict the granulation frequency in our stars. The value we found is $\sim 35 \mathrm{~d}^{-1}$, but this cannot be linked to any significant frequency or inverse timescale of the observations discussed in this paper. If granulation is the cause of the red noise in these hot $\mathrm{O}$ stars, it would therefore need to have properties that cannot simply be extrapolated from granulation in cooler-type stars.

Finally, the red noise could be caused by inhomogeneities in the stellar wind. There are various observational indicators showing that the stellar winds of early-type stars are clumped (see review by Puls et al. 2008, and references therein). The exact cause of this clumping is not clear. Intrinsic instabilities in the radiative driving mechanism have been proposed (Owocki \& Rybicki 1984), but the onset of clumping seems to happen very close to the stellar surface (Puls et al. 2006). The red noise could therefore be related to the onset of clumping at the stellar surface.

The behaviour of the early-type $\mathrm{O}$ stars (spectral types $\mathrm{O} 4-08$ ) is considerably different from that of latertype stars. When taking out the dominant binary signature of HD 47129 (O8 III/I + O7.5 V/III), a series of frequencies is found that might be caused by pulsations, but a clear red-noise component is present as well (Mahy et al. 2011). HD 46149 (O8, 
Degroote et al. 2010) does have significant frequency spacing. HD 46202 (O9 V) shows clear $\beta$ Cep-like pulsations (Briquet et al. 2011). Observations with the MOST (Microvariability and Oscillations of Stars) satellite of the O9.5 V star $\zeta$ Oph also show $\beta$ Cep-type pulsations (Walker et al. 2005). Among the early B-type stars, pulsation frequencies are easily found: Degroote et al. (2009a) present a study of 358 candidate B-star pulsators, showing numerous classical SPBs (slowly-pulsating B stars). We also note that a red-noise component is present in their spectra, but it is much more limited in frequency range than for the stars discussed here (see their Fig. 2).

The present data combined with information from the literature permit a first attempt at empirically mapping the hottest part of the instability strip for $\beta$ Cep and SPB stars. We can compare the position in the HR diagram of our stars, HD 47129 (Mahy et al. 2011), HD 46149 (Degroote et al. 2010), HD 46202 (Briquet et al. 2011) and $\zeta$ Oph (Walker et al. 2005) to theoretical predictions of the $\beta$ Cep and SPB instability strip. Zdravkov $\&$ Pamyatnykh (2008) present the theoretical strip for models up to $40 M_{\odot}$ for a metallicity $Z=0.02$. Most of the stars in our list fall into their theoretical $\beta$ Cep instability strip, but outside the SPB one. Because only a few of these stars actually show $\beta$ Cep pulsations, this suggests either that the strip is much narrower, or that the pulsations are excited for only a small fraction of the stars in the strip. Especially constraining is a group of three stars (HD 46966, HD 46202 and $\zeta$ Oph) that are close to one another in the HR diagram, with HD 46202 and $\zeta$ Oph showing $\beta$ Cep pulsations, but HD 46966 not.

A number of O-type $\beta$ Cep pulsators have also been detected in ground-based observations (e.g. Telting et al. 2006; De Cat et al. 2007; Pigulski \& Pojmański 2008). These are all late Otype stars and therefore close to the three-star group. A detailed knowledge of their astrophysical parameters would allow a better mapping of the instability strip in that part of the HR diagram. In the hotter part, we note the absence of $\beta$ Cep pulsations in the main-sequence stars discussed here. The theoretical $\beta$ Cep strip extends to the terminal age main sequence (TAMS), however, and stars more evolved than those studied here could therefore show $\beta$ Cep pulsations.

\section{Conclusions}

The CoRoT light curves of the hot O-type stars HD 46223, HD 46150 and HD 46966 were analysed using standard methods to search for pulsation frequencies. The detection of these frequencies would allow an asteroseismological interpretation. However, the results show that most of the variations of these stars are of a stochastic nature. The only significant exception to the above is the possible rotation period detected for HD 46223 and HD 46966, although these conclusions are not strong and still require confirmation. Evidence for a rotation period in the HD 46150 data is even less strong.

The periodogram of these three stars is clearly different from that of a classical pulsator such as HD 46202. Most of the power in our periodograms is not caused by pulsation, but is more correctly described by red noise. Based on the limited number of stars studied so far, a trend is suggested: the earliest $\mathrm{O}$ stars show red noise, while the later O-types have pulsational frequencies of the $\beta$ Cep type. The switch-over occurs around spectral type O8.

The specific physical cause of this noise in O-type stars is at present unclear. We point out the possibilities of subsurface convection, granulation or inhomogeneities in the wind. All three options are highly speculative and therefore await confirmation by detailed modelling. The CoRoT data of these three hot stars present interesting challenges for our understanding of the outer photosphere and inner wind of early-type stars.

Acknowledgements. We thank the CoRoT team for the acquisition and the reduction of the CoRoT data. We also thank F. Martins for making the preliminary results of his analysis available to us. We thank the anonymous referee for his/her constructive comments. L.M., E.G. and G.R. are grateful to the F.N.R.S (Belgium), the PRODEX XMM/Integral contract (Belspo), GAIA DPAC Prodex and the Communauté française de Belgique - Action de recherche concertée - A.R.C. - Académie Wallonie-Europe for their support. P.D. and C.A. acknowledge the financial support of the European Research Council under the European Community's Seventh Framework Programme (FP7/2007-2013)/ERC grant agreement No 227224 (PROSPERITY), from the Research Council of K.U. Leuven (GOA/2008/04), and from the Belgian federal science policy office (C90309: CoRoT Data Exploitation)

\section{References}

Aerts, C., Christensen-Dalsgaard, J., \& Kurtz, D. W. 2010, Asteroseismology (Dordrecht: Springer)

Auvergne, M., Bodin, P., Boisnard, L., et al. 2009, A\&A, 506, 411

Baglin, A., Auvergne, M., Barge, P., et al. 2006, in The CoRoT Mission PreLaunch Status - Stellar Seismology and Planet Finding, ed. M. Fridlund, A. Baglin, J. Lochard, \& L. Conroy (ESA, Noordwijk), ESA SP, 1306, 33

Bisiacchi, G. F., Lopez, J. A., \& Firmani, C. 1982, A\&A, 107, 252

Bonatto, C., \& Bica, E. 2009, MNRAS, 394, 2127

Briquet, M., Aerts, C., Baglin, A., et al. 2011, A\&A, 527, A112

Burderi, L. 1994, in Astronomical Society of the Pacific Conf. Ser., Vol. 61, Astronomical Data Analysis Software and Systems III, ed. D. R. Crabtree, R. J. Hanisch, \& J. Barnes (California: ASP), 353

Cantiello, M., Langer, N., Brott, I., et al. 2009, A\&A, 499, 279

Conti, P. S., \& Ebbets, D. 1977, ApJ, 213, 438

Conti, P. S., \& Leep, E. M. 1974, ApJ, 193, 113

Conti, P. S., Leep, E. M., \& Lorre, J. J. 1977, ApJ, 214, 759

De Cat, P., Briquet, M., Aerts, C., et al. 2007, A\&A, 463, 243

Degroote, P., Aerts, C., Ollivier, M., et al. 2009a, A\&A, 506, 471

Degroote, P., Briquet, M., Catala, C., et al. 2009b, A\&A, 506, 111

Degroote, P., Briquet, M., Auvergne, M., et al. 2010, A\&A, 519, A38

Diago, P. D., Gutiérrez-Soto, J., Auvergne, M., et al. 2009, A\&A, 506, 125

Dupret, M.-A., De Ridder, J., De Cat, P., et al. 2003, A\&A, 398, 677

Garmany, C. D., Conti, P. S., \& Massey, P. 1980, ApJ, 242, 1063

Gosset, E. 2007, Le calcul du niveau de signification du plus haut pic dans les périodogrammes de type Fourier par la formule de Horne-Baliunas est contreindiqué, Thèse d'Agrégation de l'Enseignement Supérieur, Seconde Thèse Annexe, University of Liège, Liège

Gosset, E., Royer, P., Rauw, G., Manfroid, J., \& Vreux, J. 2001, MNRAS, 327, 435

Grevesse, N., \& Noels, A. 1993, in Perfectionnement de l'Association Vaudoise des Chercheurs en Physique, AVCP, Lausanne, 205

Hakala, P., Ramsay, G., Wheatley, P., Harlaftis, E. T., \& Papadimitriou, C. 2004, A\&A, 420, 273

Hannan, E. J. 1980, Ann. Stat., 8, 1071

Hannan, E. J., \& Quinn, B. 1979, J. Roy. Stat. Soc. Ser. B, 41, 190

Heck, A., Manfroid, J., \& Mersch, G. 1985, A\&AS, 59, 63, (HMM)

Hensberge, H., Pavlovski, K., \& Verschueren, W. 2000, A\&A, 358, 553

Hillier, D. J., \& Miller, D. L. 1998, ApJ, 496, 407

Hiltner, W. A. 1956, ApJS, 2, 389

Howarth, I. D., \& Reid, A. H. N. 1993, A\&A, 279, 148

Huat, A.-L., Hubert, A.-M., Baudin, F., et al. 2009, A\&A, 506, 95

Kallinger, T., \& Matthews, J. M. 2010, ApJ, 711, L35

Kambe, E., Hirata, R., Ando, H., et al. 1997, ApJ, 481, 406

Kiss, L. L., Szabó, G. M., \& Bedding, T. R. 2006, MNRAS, 372, 1721

Liddle, A. R. 2007, MNRAS, 377, L74

Lomb, N. R. 1976, Ap\&SS, 39, 447

Loumos, G. L., \& Deeming, T. J. 1978, Ap\&SS, 56, 285

Lucy, L. B., \& Sweeney, M. A. 1971, AJ, 76, 544

Mahy, L., Nazé, Y., Rauw, G., et al. 2009, A\&A, 502, 937

Mahy, L., Gosset, E., Baudin, F., et al. 2011, A\&A, 525, A101

Maíz-Apellániz, J., Walborn, N. R., Galué, H. Á., \& Wei, L. H. 2004, ApJS, 151, 103

Massey, P., Johnson, K. E., \& Degioia-Eastwood, K. 1995, ApJ, 454, 151

Montgomery, M. H., \& O’Donoghue, D. 1999, Delta Scuti Star Newsletter, 13, 28

Munari, U., \& Tomasella, L. 1999, A\&AS, 137, 521

Owocki, S. P., \& Rybicki, G. B. 1984, ApJ, 284, 337 
R. Blomme et al.: Variability in the CoRoT photometry of three hot O-type stars

Perdang, J. 2009, in EAS Pub. Ser., 38, ed. M. Goupil, Z. Koláth, N. Nardetto, \& P. Kervella (Les Ulis, France: EDP), 43

Pigulski, A., \& Pojmański, G. 2008, A\&A, 477, 917

Press, W. H., Teukolsky, S. A., Vetterling, W. T., \& Flannery, B. P. 1992, Numerical recipes in FORTRAN. The art of scientific computing, 2nd edn. (Cambridge: University Press)

Puls, J., Markova, N., Scuderi, S., et al. 2006, A\&A, 454, 625

Puls, J., Vink, J. S., \& Najarro, F. 2008, A\&ARv, 16, 209

Rauw, G., De Becker, M., van Winckel, H., et al. 2008, A\&A, 487, 659

Samadi, R., Fialho, F., Costa, J. E. S., et al. 2007 [arXiv: astro-ph/0703354]

Scargle, J. D. 1982, ApJ, 263, 835

Schwarzschild, M. 1975, ApJ, 195, 137
Stanishev, V., Kraicheva, Z., Boffin, H. M. J., \& Genkov, V. 2002, A\&A, 394, 625

Telting, J. H., Schrijvers, C., Ilyin, I. V., Uytterhoeven, K., et al. 2006, A\&A, 452, 945

Templeton, M. R., \& Karovska, M. 2009, ApJ, 691, 1470

Timmer, J. \& Koenig, M. 1995, A\&A, 300, 707

Underhill, A. B., \& Gilroy, K. K. 1990, ApJ, 364, 626

Vaughan, S., Edelson, R., Warwick, R. S., \& Uttley, P. 2003, MNRAS, 345, 1271

Ventura, P., D’Antona, F., \& Mazzitelli, I. 2008, Ap\&SS, 316, 93

Walker, G. A. H., Kuschnig, R., Matthews, J. M., et al. 2005, ApJ, 623, L145

Wang, J., Townsley, L. K., Feigelson, E. D., et al. 2008, ApJ, 675, 464

Zdravkov, T., \& Pamyatnykh, A. A. 2008, J. Phys. Conf. Ser., 118, 012079 